\title{
Optimal Control of a Two-Server Heterogeneous Queueing System with Breakdowns and Constant Retrials
}

\author{
Dmitry Efrosinin ${ }^{1,2(凶)}$ and Janos Sztrik ${ }^{3}$ \\ 1 Johannes Kepler University Linz, Altenbergerstrasse 69, 4040 Linz, Austria \\ dmitry .efrosinin@jku. at \\ 2 Institute of Control Sciences, RAS, Profsoyuznaya street 65, \\ 117997 Moscow, Russia \\ 3 University of Debrecen, Egyetem tér 1, Debrecen 4032, Hungary \\ jsztrik@inf .unideb.hu \\ http://www.jku.at, http://www.ipu.ru, http://www.unideb.hu
}

\begin{abstract}
Heterogeneous servers which can differ in service speed and reliability are becoming more popular in the modelling of modern communication systems. For a two-server queueing system with one nonreliable server and constant retrial discipline we formulate an optimal allocation problem for minimizing a long-run average cost per unit of time. Using a Markov decision process formulation we prove a number of monotone properties for the increments of the dynamic-programming value function. Such properties imply the optimality of a two-level threshold control policy. This policy prescribes the usage of a less productive server if the number of customers in the queue becomes higher than a predefined level which depends on the state of a non-reliable more powerful server. We provide also a heuristic solution for the optimal threshold levels in explicit form as a function of system parameters.
\end{abstract}

Keywords: Optimal allocation $\cdot$ Markov decision process $\cdot$ Monotonicity properties $\cdot$ Threshold policy $\cdot$ Heterogeneous servers $\cdot$ Long-run average cost

\section{Introduction}

In modern communication systems the speed of data transmission in a link can interact with its reliability. In many cases this interaction is differently directed. The complementary properties of different links lead engineers to an idea to combine them in such a way that the advantages of high speed links could guarantee acceptable values of the cost and reliability characteristics. As example of a system where the data transmission links differ in speed and reliability is a Radio Frequency/Free Space Optic (RF/FSO) channel [33]. The capacity of the

D. Efrosinin - This work was funded by the Russian Foundation for Basic Research, Project No. 16-37-60072 mol_a_dk. 
RF link is limited to link throughputs in the order of tens of Mbps over distances of tens of meters. However, link availability can be maintained in most weather conditions. In contrast, the commercial FSO currently provides throughputs of several Gbps with link distances of a kilometre or more. One of the major limitations of FSO technology is the need for optical links to maintain line-of-sight and sensitivity to adverse weather conditions such as fog or heavy snowfalls. Another example of a system with combined technologies is a modern call-centre where the human operators are working together with a self-service facility which serves the calls on the basis of speech recognition methods [7].

Queueing systems with heterogeneous servers are appropriate models to describe the behaviour of communication systems with the properties mentioned above. In [14] it was proved that heterogeneous multi-server systems are superior in performance to homogeneous ones with the same total service time. This is also established in [24] in the context of manufacturing cells and systems, and it was confirmed in [30] using simulation results. In [5] a non-trivial application of a queue with heterogeneous servers to the performance evaluation of a wireless communication system is presented. The question of how to allocate the customers between heterogeneous servers in order to minimize the mean number of customers in the system has been studied by many authors. In [13] the optimality of a threshold policy was conjectured that prescribes the usage of the faster server whenever it is idle and there are customers in the system and the slower server must be used only if the queue length exceeds some prespecified threshold level. Based on a dynamic programming approach, the authors in [16] considered a similar problem and proved the optimality of a threshold policy. Some alternative proofs of this result were later given in $[11,18,34]$. The problem of optimal allocation in the system with more than two servers was studied in $[2,21,22,32]$. The allocation problem between the servers with failures was recently studied in [25], where the optimality of threshold policy and dependence of a state of an unreliable server was proved.

Analysis control procedures for queueing systems with additional cost structure with the aim of minimizing the long-run average cost per unit of time is notoriously more difficult. Some progress has been made in [20], where a model with set-up costs and hysteresis control policy was studied. The paper [23] generalizes results to the case of a multi-server system with a cost structure. A recent paper [17] introduces the optimal routing problem in two-server systems which differ in speed and quality of resolution. Multi-server retrial queueing systems have been extensively studied only for homogeneous servers. Queueing systems that combine heterogeneous servers and retrial effect were only rarely examined in research, especially in queueing theory. The authors in [28] evaluated the mean performance measures for the fastest free server allocation policy using the MOSEL performance modelling tool in the case of a finite-population multiserver retrial queueing system. In [9] the optimality of a threshold policy was proved in a queue with a constant retrial discipline, where only the customer at the head of the queue repeats an attempt to occupy the server.

The results presented here differ from those obtained in previous papers. The paper studies the structural properties of the optimal control policy for a 
two-server heterogeneous queueing system where we combine the classical model with an additional cost structure, unreliability of the server and constant retrial discipline. Our primary contribution includes a rigorous proving of the monotonicity properties of the dynamic programming value function for optimization problems with a constraint, of the threshold structure of the optimal allocation policy, evaluation of the stationary state probabilities in matrix form for the corresponding quasi-birth-and-death (QBD) process and derivation of the heuristic solution for the optimal threshold levels as a function of system parameters.

The remainder of the paper is organized as follows. Section 2 describes the Markov decision process. In Sect. 3 the optimization problem is formulated and the optimality equations for the dynamic-programming value function together with the relationship to the control policy is derived. Section 4 presents the monotonicity properties of the value function needed to establish the optimality of threshold policies. Section 5 is devoted to stationary probabilistic analysis under the given control policy. Section 6 presents the heuristic results for explicit evaluation of optimal thresholds.

\section{Mathematical Model}

Let us consider a controllable heterogeneous queueing system $M / M / 2$ with breakdowns and a constant retrial rate. Customers arrive at the system according to a Poisson process with intensity $\lambda, 0<\lambda<\infty$. The service facility consists of two heterogeneous exponential servers with intensities $\mu_{1}$ and $\mu_{2}$ with $0<\mu_{2} \leq \mu_{1}<\infty$. The customer, who is rejected to occupy one of the servers, joins a queue of orbiting customers. According to a constant retrial discipline the customer at the head of the queue retries for service after an exponential distributed time with intensity $\tau, 0<\tau<\infty$. Server 1 is assumed to be nonreliable with exponential distributed life times with intensity $\alpha$. A failure can occur in both cases, if a server is idle or busy. If the server fails, the repair starts immediately and a customer leaves this server if it was busy and joins the head of the queue. The repair time is exponential with intensity $\beta, 0<\beta<\infty$. Let us assume that a customer who gets service at a certain server, cannot change it up to the moment of leaving the system, e.g. after service completion and at a failure time. All interarrival times, intervals of successive retrials and service times, times to failure and repair times are assumed to be mutually independent. The system is supplied with a controller who gets full information about system states and can allocate customers between two servers or put it to the queue of the orbiting customers at each arrival, failure of the busy server or retrial epoch. The objective is to minimize the long-run average cost per unit of time. We propose to formulate the allocation problem as a Markov decision process and then to use the value iteration technique to prove some structural properties, e.g. threshold structure, of the optimal control policy.

Let $Q(t)$ denote the number of customers in the queue at time $t$, and $D_{j}(t)$ denote the state of server $j \in\{1,2\}$ at time $t$, where $D_{j}(t)=0$ if Server $j$ is idle, 
$D_{j}(t)=1$ if Server $j$ is busy and $D_{1}(t)=2$ if Server 1 is failed. The system states at time $t$ are described by a Markov process

$$
\{X(t)\}_{t \geq 0}=\left\{Q(t), D_{1}(t), D_{2}(t)\right\}_{t \geq 0} .
$$

The controllable model associated with a Markov process $\{X(t)\}$, which is referred to as a Markov decision process (MDP), is a five-tuple

$$
\left\{E, A,\left\{A_{0}(x), A_{b, 1}(x), A_{s}(x), x \in E, j \in\{1,2\}\right\}, \lambda_{x y}(a), c(x)\right\} .
$$

- $E$ is a state space,

$$
E=\left\{x=\left(q(x), d_{1}(x), d_{2}(x)\right) ; q(x) \geq 0, d_{1}(x) \in\{0,1,2\}, d_{2}(x) \in\{0,1\}\right\} .
$$

Further in the paper the notations $q(x)$ and $d_{j}(x)$ will be used to specify the certain components of the vector state $x \in E$. For every state $x$ we denote by $J_{0}(x), J_{1}(x)$ and $J_{2}(x)$ the sets of indices of idle, busy and failed servers,

$$
J_{0}(x)=\left\{j ; d_{j}(x)=0\right\}, J_{1}(x)=\left\{j ; d_{j}(x)=1\right\}, J_{2}(x)=\left\{1 ; d_{1}(x)=2\right\} .
$$

- $A=\{0,1,2\}$ is an action space with elements $a \in A$, where $a=0$ means "to send a customer to the queue" and $a=1,2$ - "to send a customer to Server 1 or 2". $A_{0}(x), A_{b, 1}, A_{s} \subseteq A$ denote subsets of control actions in state $x$, respectively in case of a new arrival, failure of busy Server 1 and a retrial arrival, where $A_{0}(x)=J_{0}(x) \cup\{0\}$. All other subsets can be expressed through the subset $A_{0}(x)$,

$$
\begin{aligned}
& A_{b, 1}(x)=A_{0}\left(S_{1} x\right), q(x) \geq 0,1 \in J_{1}(x), \\
& A_{s}(x)=A_{0}\left(S_{0}^{-1} x\right), q(x)>0,
\end{aligned}
$$

where $S_{0}, S_{0}^{-1}, S_{j}, S_{j}^{-1}, j \in\{1,2\}$ stand for the shift operators defined as

$$
\begin{aligned}
& S_{0} x=x+\mathbf{e}_{0}, S_{0}^{-1} x=x-\mathbf{e}_{0}, q(x)>0, \\
& S_{j} x=x+\mathbf{e}_{j}, j \in J_{0}(x) \cup J_{1}(x), S_{j}^{-1} x=x-\mathbf{e}_{j}, j \in J_{1}(x) \cup J_{2}(x),
\end{aligned}
$$

where $\mathbf{e}_{j}$ is used for the vector of dimension 3 with 1 in the $j$ th position (beginning from 0 th) and 0 elsewhere.

- $\lambda_{x y}(a)$ is a transition intensity to go from state $x$ to state $y$ under a control action $a$. It is assumed that the model is conservative, i.e.

$$
\lambda_{x y}(a) \geq 0, y \neq x, \lambda_{x x}(a)=-\lambda_{x}(a)=-\sum_{y \neq x} \lambda_{x y}(a), \lambda_{x}(a)<\infty .
$$

- $c(x)$ is an immediate cost, in state $x \in E$,

$$
c(x)=c_{0} q(x)+c_{11} 1_{\left\{1 \in J_{1}(x)\right\}}+c_{2} 1_{\left\{2 \in J_{1}(x)\right\}}+c_{12} 1_{\left\{1 \in J_{2}(x)\right\}},
$$

where $c_{0}, c_{11}, c_{12}$ and $c_{2}$ is respectively the waiting cost per unit of time for each customer in the system, the usage and repair costs for Server 1 and usage cost for Server 2. 
We will next explain how the controller chooses its actions. First the concept of a stationary policy must be introduced.

Definition 1. A stationary policy $f$ for the given model is a vector of functions

$$
f=\left(f_{0}, f_{b, 0}, f_{s}\right), \text { where } f_{\bullet}: E \rightarrow A_{\bullet}(x) .
$$

The policy $f$ specifies the control actions that must be chosen at different decision epochs whenever they occur just after an event at state $x \in E$ :

- just after an arrival, if the queue is not empty, a customer can be sent to one of the idle servers or sent to the queue;

- just after a failure of a busy server a customer from the server can be sent to the queue or to another idle server;

- just after a retrial arrival a customer can be sent from the head of the queue, if it is not empty, to an idle server.

We assume that just after any other transition no control action has to be chosen.

\section{Optimization Problem for Performance Characteristics}

For every fixed stationary policy $f$ we wish to guarantee that the process $\{X(t)\}_{t \geq 0}$ with a state-space $E$ is an irreducible, positive recurrent Markov process defined through its infinitesimal matrix $\Lambda=\left[\lambda_{x y}(f(x))\right]$. As is known [29], for an ergodic Markov process with costs the long-run average cost per unit of time (also referred to as gain) for the policy $f$ coincides with the corresponding assemble average,

$$
g^{f}=\lim _{t \rightarrow \infty} \frac{1}{t} V^{f}(x, t)=\sum_{y \in E} c(y) \pi_{y}^{f},
$$

where $V^{f}(x, t)=\int_{0}^{t} \sum_{y \in E} \mathbb{P}^{f}[X(u)=y \mid X(0)=x] c(y) d u$ denotes the total average cost up to time $t$ when the process starts in state $x$ and $\pi_{y}^{f}=\mathbb{P}^{f}[X(t)=y]$ denotes stationary probability of the process given policy $f$. The policy $f^{*}$ is said to be optimal when for any admissible policy $f$

$$
g^{f^{*}}=\min _{f} g^{f} .
$$

In many applications it is often necessary to find a policy $f^{*}$ which minimizes the long-run average cost per unit of time under the constraint on the sojourn time or the number of customers in the system (due to Little's Law), namely

$$
g^{f^{*}}=\min _{f} g^{f} \quad \text { subject to } \quad \bar{N} \leq \gamma,
$$

where $\bar{N}$ is a mean number of customers in the system. The constrained Markov decision problem can be rewritten as an unconstrained one using Lagrange multipliers, see e.g. [1,4]. The application of MDP with constraints to the problem of optimal allocation in queueing systems was illustrated e.g. in [35]. 
The optimal policy $f^{*}$ can be evaluated by means of a Howard iteration algorithm [10], which constructs a sequence of improved policies until the average cost optimal is reached. The key role in this algorithm is played by the dynamic programming value function $v: E \rightarrow \mathbb{R}_{+}$which indicates a transition effect of an initial state $x$ to the total average cost and satisfies an asymptotic relation,

$$
V^{f}(x, t)=g^{f} t+v^{f}(x)+o(1), x \in E, t \rightarrow \infty .
$$

The functions $V^{f}, v^{f}$ and $g^{f}$ further in the paper will be denoted by $V, v$ and $g$ without upper index $f$. The system will be uniformized as in [26] with

$$
\lambda+\mu_{1}+\mu_{2}+\alpha+\beta+\tau=1
$$

which can be obtained by time scaling. As is well known, the optimal policy $f$ and the optimal average cost $g$ are solutions of the optimality equation

$$
B v(x)=v(x)+g,
$$

where $B$ is the dynamic programming operator acting on value function $v$.

Theorem 1. The dynamic programming operator $B$ is defined as follows

$$
\begin{aligned}
B v(x) & =c(x)+\eta l(x)+\lambda T_{0} v(x)+\sum_{j \in J_{1}(x)} \mu_{j} T_{j} v(x) \\
& +\alpha T_{b, 0} v(x)+\alpha T_{b, 1} v(x)+\beta T_{r} v(x)+\tau T_{s} v(x) \\
& +\left(\sum_{j \notin J_{1}(x)} \mu_{j}+\beta 1_{\left\{1 \notin J_{2}(x)\right\}}\right) T_{d} v(x),
\end{aligned}
$$

where $T_{0}, T_{j}, j \in\{1,2\}, T_{b, 0}, T_{b, 1}, T_{r}, T_{s}$ and $T_{d}$ - event operators, respectively, for a new arrival, for service completion on server $j$, failure occurrence in idle or busy state, repair completion of Server 1 and retrial arrival, the last operator stands for dummy transitions,

$$
\begin{aligned}
& T_{0} v(x)=\min _{a \in A_{0}(x)} v\left(x+e_{a}\right), T_{j} v(x)=v\left(S_{j}^{-1} x\right), j \in J_{1}(x), \\
& T_{b, 0} v(x)=v\left(S_{1}^{2} x\right), 1 \in J_{0}(x), T_{b, 1} v(x)=T_{0} v\left(S_{1} x\right), 1 \in J_{1}(x), \\
& T_{r} v(x)=v\left(S_{1}^{-2} x\right), 1 \in J_{2}(x), T_{s} v(x)=\left\{\begin{array}{ll}
T_{0} v\left(S_{0}^{-1} x\right) & q(x)>0, \\
v(x) & q(x)=0 .
\end{array}, T_{d} v(x)=v(x) .\right.
\end{aligned}
$$

Proof. The optimality equation is obtained by analysing the function $V(x, t)$ in some infinitesimal interval $[t, t+d t]$. It leads to a differential equation. Applying further the limit expression for $d t \rightarrow 0$ and due to the Markov property of $\{X(t)\}_{t \geq 0}$ with asymptotic relation (12) ones get (14). Here $\eta>0$ is the Lagrange multiplier, $l(y)$ stands for the number of customers in state $y$. When $\eta$ increases, then the value of $\bar{N}$ decreases. Therefore, there exist values $\eta^{\prime}$ and $\eta^{\prime \prime}$, such that $\bar{N}\left(\eta^{\prime}\right)>\gamma$ and $\bar{N}\left(\eta^{\prime \prime}\right) \leq \gamma$, where $\eta^{\prime \prime}=\eta^{\prime}+\varepsilon$ for a small $\varepsilon \geq 0$. 
The structure of the system (14) implies for each $x \in E$ the following statement.

Corollary 1. The optimal policy $f=\left(f_{0}, f_{b, 1}, f_{s}\right)$ is defined through the value function $v(x)$ and depends only on its component $f_{0}$ for the shifted states,

$$
\begin{aligned}
& f_{0}(x)=\underset{a \in A_{0}(x)}{\arg \min }\left\{v\left(S_{a} x\right)\right\}, \\
& f_{b, 1}(x)=\underset{a \in A_{b, 1}(x)}{\arg \min }\left\{v\left(S_{a} S_{1} x\right)\right\}=f_{0}\left(S_{1} x\right), 1 \in J_{1}(x), \\
& f_{s}(x)=\underset{a \in A_{s}(x)}{\arg \min }\left\{v\left(S_{a} S_{0}^{-1} x\right)\right\}=f_{0}\left(S_{0}^{-1} x\right), q(x)>0 .
\end{aligned}
$$

Therefore, the optimal component $f_{0}$ completely defines the optimal policy $f$. To show the structural properties of the optimal policy $f$ some monotonicity properties of the increments of the value function $v(x)$ must be proved.

The relations (15) show that the structural and monotone properties of the optimal control policy $f$ can be derived by analysing the monotonicity properties of the value function $v$. Such properties for other types of controlled queues in a tandem were studied also in $[12,15,31]$. It was shown that the value function has some monotonicity properties like non-decreasing and superconvexity. To prove such inequalities it is necessary to solve (13). Since the solution of the optimality equation in analytic form is hardly available, it can be solved recursively defining $v_{n+1}=B v_{n}$ for some arbitrary initial $v_{0}$. Due to the limit relation

$$
\lim _{n \rightarrow \infty} B^{n} v_{0}(x)=v(x)
$$

we get an optimal solution for the value function. For existence and convergence solutions and optimal policies we refer to [3,25-27].

\section{Optimality of the Threshold Policy}

Now some monotonicity properties of the value function for the system under study will be presented and proved, but first we have to make a statement.

Theorem 2. The value function $v$ satisfies the conditions for any $x \in E$ : C1. Non-decreasing condition

$$
\begin{aligned}
& \text { (a) } v(x)-v\left(S_{0} x\right) \leq 0 \\
& \text { (b) } v(x)-v\left(S_{j} x\right) \leq 0, j \in\{1,2\}, d_{j}(x)=0 \\
& \text { (c) } v\left(S_{1} x\right)-v\left(S_{2} x\right) \leq 0, d_{1}(x)=d_{2}(x)=0 \\
& \text { (d) } v\left(S_{1} x\right)-v\left(S_{0} x\right) \leq 0, d_{1}(x)=0 .
\end{aligned}
$$

C2. Superconvexity condition

(a) $v\left(S_{0} x\right)-v\left(S_{2} x\right) \leq v\left(S_{0}^{2} x\right)-v\left(S_{0} S_{2} x\right), d_{1}(x) \in\{0,1,2\}, d_{2}(x)=0$,

(b) $v\left(S_{1} x\right)-v\left(S_{2} x\right) \leq v\left(S_{0} S_{1} x\right)-v\left(S_{1} S_{2} x\right), q(x)=0, d_{1}(x)=d_{2}(x)=0$. 


\section{C3. Supermodularity condition}

(a) $v\left(S_{0} x\right)-v(x) \leq v\left(S_{0} S_{2} x\right)-v\left(S_{2} x\right), d_{1}(x) \in\{0,1,2\}, d_{2}(x)=0$,

(b) $v\left(S_{1} x\right)-v(x) \leq v\left(S_{1} S_{2} x\right)-v\left(S_{2} x\right), q(x)=0, d_{1}(x)=0, d_{2}(x)=0$.

\section{C4. Convexity condition}

(a) $2 v\left(S_{0} x\right)-v(x)-v\left(S_{0}^{2} x\right) \leq 0, d_{1}(x) \in\{1,2\}, d_{2}(x)=0$,

(b) $2 v\left(S_{0} S_{2} x\right)-v\left(S_{2} x\right)-v\left(S_{0}^{2} S_{2} x\right) \leq 0, d_{1}(x) \in\{1,2\}, d_{2}(x)=0$,

(c) $2 v\left(S_{1} x\right)-v(x)-v\left(S_{0} S_{1} x\right) \leq 0, d_{1}(x)=0, d_{2}(x)=0$.

Statement 1. The inequality (C4-a) directly follows by summing up the conditions (C2-a) and (C3-a), the inequality (C4-b) - by summing up the conditions (C2-a) and (C3-a) in state $x$ and $S_{0} x$ respectively and (C4-c) - by summing up the conditions (C2-b) and (C3-b), which are the boundary conditions for the state $x=(0,0,0)$. The name of condition (C3) is borrowed from [6].

Statement 2. (C1-c) can be explicitly proved only under assumption $c_{11}-c_{2} \leq 0$ and $(\mathrm{C} 1-\mathrm{d})-$ under assumption $c_{11}-c_{0} \leq 0$, which are of course too strong. We assume that for conditions $(\mathrm{C} 1)-(\mathrm{C} 4)$ the expression $(26)$ must be positive.

Due to lack of space we demonstrate the proof only for the properties (C1-c) and $(\mathrm{C} 2-\mathrm{a})$. The rest of the inequalities can be proved in a similar way.

Proof (Condition C1). The proof is by induction on $n$ in $v_{n}$. Let us define $v_{0}(x)=0$ for all states $x \in E$. This function obviously satisfies the conditions $(\mathrm{C} 1)-(\mathrm{C} 3)$. Now, we assume $(\mathrm{C} 1)-(\mathrm{C} 3)$ for the function $v_{n}(x), x \in E$, and some $n \in \mathbb{N}$. One has to prove that $v_{n+1}(x)$ satisfies the proposed conditions as well.

(C1-c). Let us consider the inequality (C1-c). For this increment we get,

$$
\begin{aligned}
& v_{n+1}\left(S_{1} x\right)-v_{n+1}\left(S_{2} x\right)=c\left(S_{1} x\right)-c\left(S_{2} x\right)+\eta\left(l\left(S_{1} x\right)-l\left(S_{2} x\right)\right) \\
& +\lambda\left[T_{0} v_{n}\left(S_{1} x\right)-T_{0} v_{n}\left(S_{2} x\right)\right] \\
& +\mu_{1}\left[T_{1} v_{n}\left(S_{1} x\right)-T_{d} v_{n}\left(S_{2} x\right)\right] \\
& +\mu_{2}\left[T_{d} v_{n}\left(S_{1} x\right)-T_{2} v_{n}\left(S_{2} x\right)\right] \\
& +\alpha\left[T_{b, 1} v_{n}\left(S_{1} x\right)-T_{b, 0} v_{n}\left(S_{2} x\right)\right] \\
& +\tau\left[T_{s} v_{n}\left(S_{1} x\right)-T_{s} v_{n}\left(S_{2} x\right)\right] \\
& +\beta\left[T_{d} v_{n}\left(S_{1} x\right)-T_{d} v_{n}\left(S_{2} x\right)\right] \leq 0 .
\end{aligned}
$$

The term (I) is equal to $c_{11}-c_{2} \leq 0$, which is true by assumption. The term (II)

$$
\text { (II) }=T_{0} v_{n}\left(S_{1} x\right)-v_{n}\left(S_{1} S_{2} x\right) \leq v_{n}\left(S_{1} S_{2} x\right)-v_{n}\left(S_{1} S_{2} x\right)=0
$$

by virtue of (C1-c) and (C1-d). Now we join the terms (III) and (IV),

$$
\begin{aligned}
(I I I)+(I V) & =\mu_{2}\left[T_{d} v_{n}\left(S_{1} x\right)-T_{2} v_{n}\left(S_{2} x\right)\right]-\mu_{1}\left[T_{d} v_{n}\left(S_{2} x\right)-T_{1} v_{n}\left(S_{1} x\right)\right] \\
& =\mu_{2}\left[v_{n}\left(S_{1} x\right)-v_{n}(x)\right]-\mu_{1}\left[v_{n}\left(S_{2} x\right)-v_{n}(x)\right] \\
& =\mu_{1} \mu_{2}\left[\frac{v_{n}\left(S_{1} x\right)-v_{n}(x)}{\mu_{1}}-\frac{v_{n}\left(S_{2} x\right)-v_{n}(x)}{\mu_{2}}\right] \leq 0,
\end{aligned}
$$


for $q(x)>0$ due to $\mu_{1} \geq \mu_{2}$ and (C1-b). The term (V) is non-positive,

$$
(\mathrm{V})=T_{0} v_{n}\left(S_{1}^{2} x\right)-v_{n}\left(S_{1}^{2} S_{2} x\right) \leq v_{n}\left(S_{1}^{2} S_{2} x\right)-v_{n}\left(S_{1}^{2} S_{2} x\right)=0 .
$$

For the term (VI) we have

$$
\begin{aligned}
(\mathrm{VI}) & =T_{0} v_{n}\left(S_{0}^{-1} S_{1} x\right)-T_{0} v_{n}\left(S_{0}^{-1} S_{2} x\right)=T_{0} v_{n}\left(S_{0}^{-1} S_{1} x\right)-v_{n}\left(S_{0}^{-1} S_{1} S_{2} x\right) \\
& \leq v_{n}\left(S_{0}^{-1} S_{1} S_{2} x\right)-v_{n}\left(S_{0}^{-1} S_{1} S_{2} x\right)=0, \text { for } q(x)>0, \\
(\mathrm{VI}) & =v_{n}\left(S_{1} x\right)-v_{n}\left(S_{2} x\right) \leq 0, \text { for } q(x)=0,
\end{aligned}
$$

by (C1-c). The term (VII) is non-positive according to (C1-c). If (C1) holds for $v_{n}(x)$ then it holds by induction for any $n$ and by (16) for the function $v(x)$.

Proof (Condition C2). The proof is made by induction on $n$ in $v_{n}$. Let us define $v_{0}(x)=0$ for all states $x \in E$. It is clear that this function satisfies condition (C2). Now suppose that properties (C1)-(C4) hold for $v_{n}, n \in \mathbb{N}$. Now we prove that it holds for $n+1$ as well.

(C2-a). In this case for the function $v_{n}(x)$ holds the following inequality

$$
\begin{aligned}
& v_{n+1}\left(S_{0} x\right)-v_{n+1}\left(S_{2} x\right)-v_{n+1}\left(S_{0}^{2} x\right)+v_{n+1}\left(S_{0} S_{2} x\right) \\
& =c\left(S_{0} x\right)-c\left(S_{2} x\right)-c\left(S_{0}^{2} x\right)+c\left(S_{0} S_{2} x\right) \\
& +\eta\left(l\left(S_{0} x\right)-l\left(S_{2} x\right)-l\left(S_{0}^{2} x\right)+l\left(S_{0} S_{2} x\right)\right) \\
& +\lambda\left[T_{0} v_{n}\left(S_{0} x\right)-T_{0} v_{n}\left(S_{2} x\right)-T_{0} v_{n}\left(S_{0}^{2} x\right)+T_{0} v_{n}\left(S_{0} S_{2} x\right)\right] \\
& +\mu_{1}\left[T_{1} v_{n}\left(S_{0} x\right)-T_{1} v_{n}\left(S_{2} x\right)-T_{1} v_{n}\left(S_{0}^{2} x\right)+T_{1} v_{n}\left(S_{0} S_{2} x\right)\right] \\
& +\alpha\left[T_{b, 1} v_{n}\left(S_{0} x\right)-T_{b, 1} v_{n}\left(S_{2} x\right)-T_{b, 1} v_{n}\left(S_{0}^{2} x\right)+T_{b, 1} v_{n}\left(S_{0} S_{2} x\right)\right] \\
& +\beta\left[T_{r} v_{n}\left(S_{0} x\right)-T_{r} v_{n}\left(S_{2} x\right)-T_{r} v_{n}\left(S_{0}^{2} x\right)+T_{r} v_{n}\left(S_{0} S_{2} x\right)\right] \\
& +\mu_{2}\left[T_{d} v_{n}\left(S_{0} x\right)-T_{2} v_{n}\left(S_{2} x\right)-T_{d} v_{n}\left(S_{0}^{2} x\right)+T_{2} v_{n}\left(S_{0} S_{2} x\right)\right] \\
& +\tau\left[T_{s} v_{n}\left(S_{0} x\right)-T_{s} v_{n}\left(S_{2} x\right)-T_{s} v_{n}\left(S_{0}^{2} x\right)+T_{s} v_{n}\left(S_{0} S_{2} x\right)\right] \\
& +\left(\left(\alpha+\mu_{1}\right) 1_{\left\{d_{1}(x)=2\right\}}+\beta 1_{\left.\left\{d_{1}(x)=1\right\}\right)}\right) \\
& \times\left[T_{d} v_{n}\left(S_{0} x\right)-T_{d} v_{n}\left(S_{2} x\right)-T_{d} v_{n}\left(S_{0}^{2} x\right)+T_{d} v_{n}\left(S_{0} S_{2} x\right)\right] \leq 0 .
\end{aligned}
$$

The term (I) is obviously 0 . We show that the term (II) is non-positive. To perform it let us consider the following two subcases. If $f_{0}\left(S_{2} x\right)=f\left(S_{0}^{2} x\right)=0$, then

$$
\begin{aligned}
(\mathrm{II}) & =T_{0} v_{n}\left(S_{0} x\right)-v_{n}\left(S_{0} S_{2} x\right)-v_{n}\left(S_{0}^{3} x\right)+T_{0} v_{n}\left(S_{0} S_{2} x\right) \\
& \leq v_{n}\left(S_{0}^{2} x\right)-v_{n}\left(S_{0} S_{2} x\right)-v_{n}\left(S_{0}^{3} x\right)+v_{n}\left(S_{0}^{2} S_{2} x\right) \leq 0
\end{aligned}
$$

due to (C2-a) in state $S_{0} x$. If $f_{0}\left(S_{2} x\right)=0$ and $f\left(S_{0}^{2} x\right)=2$, then

$$
\begin{aligned}
(\mathrm{II}) & =T_{0} v_{n}\left(S_{0} x\right)-v_{n}\left(S_{0} S_{2} x\right)-v_{n}\left(S_{0}^{2} S_{2} x\right)+T_{0} v_{n}\left(S_{0} S_{2} x\right) \\
& \leq v_{n}\left(S_{0} S_{2} x\right)-v_{n}\left(S_{0} S_{2} x\right)-v_{n}\left(S_{0}^{2} S_{2} x\right)+v_{n}\left(S_{0}^{2} S_{2} x\right)=0 .
\end{aligned}
$$


The term (III) satisfies

$$
(\mathrm{III})=v_{n}\left(S_{0} S_{1}^{-1} x\right)-v_{n}\left(S_{1}^{-1} S_{2} x\right)-v_{n}\left(S_{0}^{2} S_{1}^{-1} x\right)+v_{n}\left(S_{0} S_{1}^{-1} S_{2} x\right) \leq 0,
$$

which follows from the inequalities (C2-a). Let us consider the term (IV). We have

$$
(\mathrm{IV})=T_{0} v_{n}\left(S_{0} S_{1}^{2} x\right)-T_{0} v_{n}\left(S_{1}^{2} S_{2} x\right)-T_{0} v_{n}\left(S_{0}^{2} S_{1}^{2} x\right)+T_{0} v_{n}\left(S_{0} S_{1}^{2} S_{2} x\right) \leq 0,
$$

with respect to the property of the operator $T_{0}$ applied to $(\mathrm{C} 2-\mathrm{a})$ in state $S_{1}^{2} x$. The term (V) is of the form

$$
(\mathrm{V})=v_{n}\left(S_{0} S_{1}^{-2} x\right)-v_{n}\left(S_{1}^{-2} S_{2} x\right)-v_{n}\left(S_{0}^{2} S_{1}^{-2} x\right)+v_{n}\left(S_{0} S_{1}^{-2} S_{2} x\right) \leq 0,
$$

which follows from the inequality (C2-a) in state $S_{1}^{-2} x$ for $d_{1}(x)=2$. For the term (VI) we have

$$
(\mathrm{VI})=v_{n}\left(S_{0} x\right)-v_{n}(x)-v_{n}\left(S_{0}^{2} x\right)+v_{n}\left(S_{0} x\right)=2 v_{n}\left(S_{0} x\right)-v_{n}(x)-v_{n}\left(S_{0}^{2} x\right) \leq 0,
$$

by (C4-a). The term (VII) is non-positive for $q(x)>0$ due to the property of the operator $T_{s}$ and $T_{0}$ applied to the state $S_{0}^{-1} x$ in (C2-a),

$$
(\mathrm{VII})=T_{0} v_{n}(x)-T_{0} v_{n}\left(S_{0}^{-1} S_{2} x\right)-T_{0} v_{n}\left(S_{0} x\right)+T_{0} v_{n}\left(S_{2} x\right) \leq 0 .
$$

For $q(x)=0$ we get

$$
(\mathrm{VII})=T_{0} v_{n}(x)-v_{n}\left(S_{2} x\right)-T_{0} v_{n}\left(S_{0} x\right)+T_{0} v_{n}\left(S_{2} x\right) .
$$

If $f_{0}\left(S_{0} x\right)=0$, then we have

$$
\begin{aligned}
& (\mathrm{VII})=T_{0} v_{n}(x)-v_{n}\left(S_{2} x\right)-v_{n}\left(S_{0}^{2} x\right)+T_{0} v_{n}\left(S_{2} x\right) \\
& \leq v_{n}\left(S_{0} x\right)-v_{n}\left(S_{2} x\right)-v_{n}\left(S_{0}^{2} x\right)+v_{n}\left(S_{0} S_{2} x\right) \leq 0
\end{aligned}
$$

as in (C2-a). If $f_{0}\left(S_{0} x\right)=2$, then

$$
\begin{aligned}
& (\mathrm{VII})=T_{0} v_{n}(x)-v_{n}\left(S_{2} x\right)-v_{n}\left(S_{0} S_{2} x\right)+T_{0} v_{n}\left(S_{2} x\right) \\
& \leq v_{n}\left(S_{2} x\right)-v_{n}\left(S_{2} x\right)-v_{n}\left(S_{0} S_{2} x\right)+v_{n}\left(S_{0} S_{2} x\right)=0 .
\end{aligned}
$$

The last term (VIII) is non-positive by (C2-a).

Hence, we conclude, by taking the limit $n \rightarrow \infty$, that the value function $v(x)$ preserves the conditions $(\mathrm{C} 2)$.

Corollary 2. The optimal allocation policy is of threshold type. There exists a two-level threshold policy $f^{*}=\left(q_{1}^{*}, q_{2}^{*}\right)$ such that

1. Server 1 must be used whenever it is free while a customer tries to get service at a service facility.

2. Server 2 must be used whenever it is free, Server 1 is busy or failed, a customer tries to get service and the queue length $q(x)$ upon arrival in state $x$ satisfies $q(x) \geq q_{k}^{*}-1$ if $d_{1}(x)=k, k \in\{1,2\}$.

Proof. The statement follows directly from the definition (15) of the control policy $f(x)$ and monotonicity properties of the value function (C1-c), (C1-d) and (C2-a) given in Theorem 2. 


\section{$5 \quad$ Stationary State Probabilities}

Now we fix the threshold policy $f=\left(q_{1}, q_{2}\right)$. The possible states of the system at any time are presented by the vector state $x=\left(q, d_{1}, d_{2}\right) \in E$. Let us define the levels $\mathbf{0}, \mathbf{1}, \mathbf{2}, \ldots$ as a set (block) of states,

$$
\mathbf{q}=\{(q, 0,0),(q, 1,0),(q, 2,0),(q, 0,1),(q, 1,1),(q, 2,1)\}
$$

for $q \geq 0$. Let the row-vector of the stationary state probabilities $\pi$ be partitioned as $\boldsymbol{\pi}=\left(\boldsymbol{\pi}_{0}, \boldsymbol{\pi}_{1}, \boldsymbol{\pi}_{2}, \ldots\right)$, where $\boldsymbol{\pi}_{q}=\left\{\pi_{(q, 0,0)}, \pi_{(q, 1,0)}, \pi_{(q, 2,0)}, \pi_{(q, 0,1)}\right.$, $\left.\pi_{(q, 1,1)}, \pi_{(q, 2,1)}\right\}$. Let us denote by $\mathbf{e}_{j}$ the vector of dimension 6 with 1 in the $j$ th position (beginning from 0 th) and 0 elsewhere and by e the unit vector of the same dimension.

Theorem 3. If the stability condition holds, the vector $\boldsymbol{\pi}$ exists and is a unique solution of the system $\boldsymbol{\pi} \Lambda=\mathbf{0}, \boldsymbol{\pi} \mathbf{e}=1$, where $\Lambda:=\Lambda\left(q_{1}, q_{2}\right)$ specifies a three diagonal block infinitesimal matrix of $\{X(t)\}_{t \geq 0}$, which is of the $Q B D$ type:

$$
\begin{aligned}
\Lambda\left(q_{1}, q_{2}\right) & =\operatorname{diag}(Q_{1,0}, \underbrace{Q_{1,1}, \ldots, Q_{1,1}}_{q_{2}-2}, Q_{1,2}, \underbrace{Q_{1,3}, \ldots, Q_{1,3}}_{q_{1}-q_{2}-1}, Q_{1,4}, Q_{1,5}, \ldots) \\
& +\operatorname{diag}^{+}(\underbrace{Q_{0,1}, \ldots, Q_{0,1}}_{q_{2}-2}, \underbrace{Q_{0,2}, \ldots, Q_{0,2}}_{q_{1}-q_{2}-1}, Q_{0,3}, \ldots) \\
& +\operatorname{diag}^{-}(\underbrace{Q_{2,1}, \ldots, Q_{2,1}}_{q_{2}-2}, \underbrace{Q_{2,2}, \ldots, Q_{2,4}}_{q_{1}-q_{2}-1}, Q_{2,3}, \ldots) .
\end{aligned}
$$

with $\left(Q_{1,0}+Q_{0,1}\right) \mathbf{e}=\left(Q_{2,1}+Q_{1,1}+Q_{0,1}\right) \mathbf{e}=\left(Q_{2,1}+Q_{1,2}+Q_{0,2}\right) \mathbf{e}=\left(Q_{2,2}+\right.$ $\left.Q_{1,3}+Q_{0,2}\right) \mathbf{e}=\left(Q_{2,2}+Q_{1,4}+Q_{0,3}\right) \mathbf{e}=\left(Q_{2,3}+Q_{1,5}+Q_{0,3}\right) \mathbf{e}=\mathbf{0}$.

Blocks $Q_{1, n}, n=\overline{0,5}$, include outgoing intensities for the certain block state,

$Q_{1,0}=\left(\begin{array}{cccccc}-(\lambda+\alpha) & \lambda & \alpha & 0 & 0 & 0 \\ \mu_{1} & -\left(\lambda+\mu_{1}+\alpha\right) & 0 & 0 & 0 & 0 \\ \beta & 0 & -(\lambda+\beta) & 0 & 0 & \alpha \\ \mu_{2} & 0 & 0 & -\left(\lambda+\mu_{2}+\alpha\right) & \lambda & 0 \\ 0 & \mu_{2} & 0 & \mu_{1} & -\left(\lambda+\mu_{1}+\mu_{2}+\alpha\right) & 0 \\ 0 & 0 & \mu_{2} & \beta & 0 & -\left(\lambda+\mu_{2}+\beta\right)\end{array}\right)$,

$Q_{1,1}=Q_{1,0}+\tau\left(\mathbf{e}_{0} \otimes \mathbf{e}_{0}^{\prime}+\mathbf{e}_{3} \otimes \mathbf{e}_{3}^{\prime}\right), Q_{1,2}=Q_{1,1}+\lambda \mathbf{e}_{2} \otimes \mathbf{e}_{5}^{\prime}+\alpha \mathbf{e}_{1} \otimes \mathbf{e}_{5}^{\prime}$,

$Q_{1,3}=Q_{1,2}+\tau \mathbf{e}_{2} \otimes \mathbf{e}_{2}^{\prime}, Q_{1,4}=Q_{1,3}+\lambda \mathbf{e}_{1} \otimes \mathbf{e}_{4}^{\prime}, Q_{1,5}=Q_{1,4}+\tau \mathbf{e}_{1} \otimes \mathbf{e}_{1}^{\prime}$.

Blocks $Q_{0, n}, n=\overline{1,3}$, include the incoming intensities from the lower level,

$$
\begin{aligned}
& Q_{0,1}=\operatorname{diag}(0, \lambda, \lambda, 0, \lambda, \lambda)+\operatorname{diag}^{+}(0, \alpha, 0,0, \alpha) \\
& Q_{0,2}=Q_{0,1}-\lambda \mathbf{e}_{2} \otimes \mathbf{e}_{2}^{\prime}-\alpha \mathbf{e}_{1} \otimes \mathbf{e}_{2}^{\prime}, Q_{0,3}=Q_{0,2}-\lambda \mathbf{e}_{1} \otimes \mathbf{e}_{1}^{\prime} .
\end{aligned}
$$

Blocks $Q_{2, n}, n=\overline{1,3}$, include the incoming intensities from the upper level,

$$
Q_{2,1}=\operatorname{diag}^{+}(\tau, 0,0, \tau, 0), Q_{2,2}=Q_{2,1}+\tau \mathbf{e}_{2} \otimes \mathbf{e}_{5}^{\prime}, Q_{2,3}=Q_{2,2}+\tau \mathbf{e}_{1} \otimes \mathbf{e}_{4}^{\prime} .
$$


For the given QBD process to be stable, it is necessary ([19], Theorem 3.1.1, p.82) that $\mathbf{p} Q_{0,3} \mathbf{e}<\mathbf{p} Q_{2,3} \mathbf{e}$, where $\mathbf{p}$ is an invariant probability, which can be evaluated from $\mathbf{p}\left(Q_{0,3}+Q_{1,5}+Q_{2,3}\right)=\mathbf{0}$ and $\mathbf{p e}=1$.

Theorem 4. The macro-vectors $\boldsymbol{\pi}_{q}, q \geq 0$, satisfy the relations

$$
\begin{aligned}
& \boldsymbol{\pi}_{q}=\boldsymbol{\pi}_{q_{1}} \prod_{j=1}^{q_{1}-q} M_{q_{1}-j}, q=\overline{0, q_{1}-1}, \\
& \boldsymbol{\pi}_{q}=\boldsymbol{\pi}_{q_{1}} R^{q-q_{1}}, q \geq q_{1},
\end{aligned}
$$

where matrices $M_{q}$ are of the form,

$$
\begin{aligned}
& M_{0}=-Q_{2,1} Q_{1,0}^{-1}, M_{q}=-Q_{2,1}\left(M_{q-1} Q_{0,1}+Q_{1,1}\right)^{-1}, q=\overline{1, q_{2}-2}, \\
& M_{q_{2}-1}=-Q_{2,2}\left(M_{q_{2}-2} Q_{0,1}+Q_{1,2}\right)^{-1}, M_{q_{1}-1}=-Q_{2,3}\left(M_{q_{1}-2} Q_{0,2}+Q_{1,4}\right)^{-1}, \\
& M_{q}=-Q_{2,2}\left(M_{q-1} Q_{0,2}+Q_{1,3}\right)^{-1}, q=\overline{q_{2}, q_{1}-2} .
\end{aligned}
$$

The vector $\boldsymbol{\pi}_{q_{1}}$ is a unique solution of the system of equations

$$
\begin{aligned}
& \boldsymbol{\pi}_{q_{1}}\left[\sum_{q=0}^{q_{1}-1} \prod_{j=1}^{q_{1}-q} M_{q_{1}-j}+(I-R)^{-1}\right] \mathbf{e}=1, \\
& \boldsymbol{\pi}_{q_{1}}\left(M_{q_{1}-1} Q_{0,3}+Q_{1,5}+R Q_{2,3}\right)=\mathbf{0} .
\end{aligned}
$$

Matrix $R$ is a minimum non-negative solution of the matrix quadratic equation,

$$
R^{2} Q_{2,3}+R Q_{1,5}+Q_{0,3}=0 .
$$

Proof. The results for boundary blocks, if $q<q_{1}$, follows by recursive solution of the balance equation after routine block identification. For $q \geq q_{1}$ we have a matrix geometric solution as shown in [19].

Corollary 3. Optimization problem consists in minimizing of the function

$$
g=c_{0} \bar{Q}+c_{11} \bar{U}_{1}+c_{2} \bar{U}_{2}+c_{12} \bar{B}
$$

subject to $\bar{N} \leq \gamma$. Here $\bar{Q}$ is the mean number of customers in the queue,

$$
\bar{Q}=\left[\sum_{q=0}^{q_{1}-1} q \boldsymbol{\pi}_{q}+\boldsymbol{\pi}_{q_{1}}\left(R+q_{1}(I-R)\right)(I-R)^{-2}\right] \mathbf{e},
$$

$\bar{U}_{j}=\mathbb{P}\left[D_{j}(t)=1\right]$ stands for the utilization of the Server $j$,

$$
\bar{U}_{j}=\left[\sum_{q=0}^{q_{1}-1} \boldsymbol{\pi}_{q}+\boldsymbol{\pi}_{q_{1}}(I-R)^{-1}\right]\left(\mathbf{e}_{1} 1_{\{j=1\}}+\left(\mathbf{e}_{3}+\mathbf{e}_{5}\right) 1_{\{j=2\}}+\mathbf{e}_{4}\right),
$$

$\bar{B}=\mathbb{P}\left[D_{1}(t)=2\right]$ is a failure index of the Server 1 ,

$$
\bar{B}=\left[\sum_{q=0}^{q_{1}-1} \boldsymbol{\pi}_{q}+\boldsymbol{\pi}_{q_{1}}(I-R)^{-1}\right]\left(\mathbf{e}_{2}+\mathbf{e}_{5}\right),
$$

and $\bar{N}$ is the mean number of customers in the system, $\bar{N}=\bar{Q}+\bar{U}_{1}+\bar{U}_{2}$. 


\section{Heuristic Solution for Optimal Thresholds}

The Howard iteration algorithm allows us to evaluate numerically the optimal threshold levels. This method has weak spots connected with a necessity to solve the system of $6 \mathrm{~N}$ linear equations for any fixed set of values of the system parameters. Here $N$ specifies a truncation parameter to transform an infinite buffer system to a finite equivalent. A direct analytic minimization of the function $g\left(q_{1}, q_{2}\right)$ is infeasible and numerical evaluation requires evaluation of the corresponding matrices $M_{q}, q=\overline{0, q_{1}-1}$ for each pair $\left(q_{1}, q_{2}\right)$.

Let us consider first the equivalent scheduling problem where is assumed that there are customers in the system and the problem consists in allocation between the servers until the system becomes empty with the aim of the total expected cost minimization. That means that in the original system the arrival rate $\lambda$ must be set to be 0 .

Theorem 5. The optimal thresholds $q_{k}^{*}, k \in\{1,2\}$, for the scheduling problem satisfy the relations,

$$
\begin{aligned}
& q_{k}^{*}=\max \left\{1,\left\lfloor\frac{1}{c_{0} A_{k}}\left[\frac{c_{2}}{\mu_{2}}-\frac{c_{11}}{\mu_{1}}-c_{0} B_{k}-c_{12} C_{k}\right]\right\rfloor\right\}, \text { where } \\
& A_{1}=\frac{(\alpha+\beta)\left(\alpha+\mu_{1}+\tau\right)}{\mu_{1} \beta \tau}, A_{2}=A_{1}+\frac{\alpha+\beta+\tau}{\beta \tau}, \\
& B_{1}=B_{2}=\frac{\alpha(\alpha+\beta+\tau)}{\mu_{1} \beta \tau}, C_{1}=\frac{\alpha\left(\alpha+\mu_{1}+\tau\right)}{\mu_{1} \beta \tau}, C_{2}=C_{1}+\frac{\alpha+\tau}{\beta \tau} .
\end{aligned}
$$

Proof. Let us consider the case $\lambda=0$. Due to the threshold structure of the control policy $f$, we can calculate the total average cost $V(x)$ incurred by the customers presented in the system until it becomes empty given initial state $x$. Assuming the known values of $\left(q_{1}, q_{2}\right)$ after some algebra we get for the state $x=(0,0,0)$,

$$
\begin{aligned}
V\left(S_{0}^{q} S_{1} x\right) & =\frac{c_{11}}{\mu_{1}}+c_{0} q \frac{(\alpha+\beta)\left(\alpha+\mu_{1}+\tau\right)}{\mu_{1} \beta \tau}+c_{0} \frac{\alpha(\alpha+\beta+\tau)}{\mu_{1} \beta \tau} \\
& +c_{12} \frac{\alpha\left(\alpha+\mu_{1}+\tau\right)}{\mu_{1} \beta \tau}+V\left(S_{0}^{q-1} S_{1} x\right), \\
V\left(S_{0}^{q+1} S_{1}^{2} x\right) & =\frac{c_{11}}{\mu_{1}}+c_{0} q\left[\frac{(\alpha+\beta)\left(\alpha+\mu_{1}+\tau\right)}{\mu_{1} \beta \tau}+\frac{\alpha+\beta+\tau}{\beta \tau}\right]+c_{0} \frac{\alpha(\alpha+\beta+\tau)}{\mu_{1} \beta \tau} \\
& +c_{12}\left[\frac{\alpha\left(\alpha+\mu_{1}+\tau\right)}{\mu_{1} \beta \tau}+\frac{\alpha+\tau}{\tau \beta}\right]+V\left(S_{0}^{q-1} S_{1} x\right) .
\end{aligned}
$$

By successive substitution of expressions for $v\left(S_{0}^{q} S_{1} x\right)$ we obtain

$$
\begin{aligned}
V\left(S_{0}^{q} S_{1} x\right) & =\frac{c_{11}}{\mu_{1}}+c_{0} \frac{q(q+1)}{2} \frac{(\alpha+\beta)\left(\alpha+\mu_{1}+\tau\right)}{\mu_{1} \beta \tau} \\
& +(q+1)\left[c_{0} \frac{\alpha(\alpha+\beta+\tau)}{\mu_{1} \beta \tau}+c_{12} \frac{\alpha\left(\alpha+\mu_{1}+\tau\right)}{\mu_{1} \beta \tau}\right] \\
& =\frac{c_{11}}{\mu_{1}}+c_{0} \frac{q(q+1)}{2} A_{1}+(q+1)\left[c_{0} B_{1}+c_{12} C_{1}\right]
\end{aligned}
$$




$$
\begin{aligned}
V\left(S_{0}^{q} S_{1}^{2} x\right) & =\frac{c_{11}}{\mu_{1}}+c_{0} \frac{q(q+1)}{2}\left[\frac{(\alpha+\beta)\left(\alpha+\mu_{1}+\tau\right)}{\mu_{1} \beta \tau}+\frac{\alpha+\beta+\tau}{\beta \tau}\right] \\
& +(q+1)\left[c_{0} \frac{\alpha(\alpha+\beta+\tau)}{\mu_{1} \beta \tau}+c_{12}\left[\frac{\alpha\left(\alpha+\mu_{1}+\tau\right)}{\mu_{1} \beta \tau}+\frac{\alpha+\tau}{\tau \beta}\right]\right] \\
& =\frac{c_{11}}{\mu_{1}}+c_{0} \frac{q(q+1)}{2} A_{2}+(q+1)\left[c_{0} B_{2}+c_{12} C_{2}\right] .
\end{aligned}
$$

When the orbit has reached the level $q_{1}$ or $q_{2}$, it becomes optimal to use the second server. Then the following inequalities must hold,

$$
\begin{aligned}
& V\left(S_{0}^{q-1} S_{1} S_{2} x\right)=\frac{c_{2}}{\mu_{2}}+V\left(S_{0}^{q-1} S_{1} x\right) \leq V\left(S_{0}^{q} S_{1} x\right) \\
& V\left(S_{0}^{q-1} S_{1}^{2} S_{2} x\right)=\frac{c_{2}}{\mu_{2}}+V\left(S_{0}^{q-1} S_{1} x\right) \leq V\left(S_{0}^{q} S_{1}^{2} x\right) .
\end{aligned}
$$

Solving the last two inequalities using the notations (24) for the expressions (25) we get the relations (23) for the optimal thresholds $\left(q_{1}^{*}, q_{2}^{*}\right)$.

In [8] the heuristic solution was obtained for the ordinary $M / M / 2$ queue. Substituting in this relation the mean service $\frac{1}{\mu_{1}}$ by the effective mean service time of the model under study $A_{k}$, taking into account the cost structure and form of the scheduling threshold levels, we can make the following conjecture.

Conjecture 1. The optimal threshold $q_{k}^{*}, k \in\{1,2\}$, for the case $\lambda>0$ can be calculated approximately by

$$
\begin{aligned}
q_{k}^{*} \approx \hat{q}_{k}^{*} & =\max \left\{1,\left\lfloor\frac{1}{c_{0} A_{k}}\left[\frac{c_{2}}{\mu_{2}} F_{k}-\frac{c_{11}}{\mu_{1}}-c_{0} B_{k}-c_{12} C_{k}\right]\right\rfloor\right\}, \text { where } \\
F_{k} & =\frac{1-\lambda A_{k}+\sqrt{\left(1-\lambda A_{k}\right)^{2}+4 \lambda \mu_{2} A_{k}^{2}}}{2}
\end{aligned}
$$

Statement 3. The threshold levels defined by (23) satisfy the inequalities

$$
\begin{aligned}
& \frac{1}{c_{0} A_{k}}\left[\frac{c_{2}}{\mu_{2}}\left(1-\lambda A_{k}\right)-\frac{c_{11}}{\mu_{1}}-c_{0} B_{k}-c_{12} C_{k}\right] \leq \hat{q}_{k} \\
& \leq \frac{1}{c_{0} A_{k}}\left[\frac{c_{2}}{\mu_{2}}-\frac{c_{11}}{\mu_{1}}-c_{0} B_{k}-c_{12} C_{k}\right], \text { for } \mu_{2} \leq \frac{1}{A_{k}}
\end{aligned}
$$

Proof. The left inequality of (28) follows directly from

$$
F_{k} \geq \frac{1-\lambda A_{k}+\sqrt{\left(1-\lambda A_{k}\right)^{2}}}{2}=1-\lambda A_{k} .
$$

To prove the inequality at the right hand side we show that $F_{k} \leq 1$. By solving this inequality using simple algebraic manipulations we get $\mu_{2} \leq A_{k}^{-1}$, where $A_{k}$ represents the mean effective service time of the customer.

Statement 4. Because of the restricted volume space we have skipped numerical examples and make here only two general observations: 
1. The optimal threshold policy can be up to $25 \%$ superior in performance compared with policies like Fastest Free Server or Random Server Selection.

2. The difference between the real and heuristic policies does not exceed $1.5 \%$ of the performance value.

\section{Conclusion}

In this paper we have studied a dynamic allocation problem for a two-server heterogeneous queueing system. The more productive server is unreliable and subject to breakdowns whereas the less productive is absolutely reliable. Under some assumption about the costs it was proved that the optimality of a threshold control policy depends on the state of the unreliable server. We expect that this result can be generalized to the multi-server retrial queue where all servers are unreliable and to the limited case where retrial intensity tends to infinity. But in this case, as for the ordinary $M / M / K$ queue, the optimal threshold levels may depend also on the states of slower servers although this influence is very negligible.

\section{References}

1. Altman, E.: Constrained Markov Decision Processes. Chapman and Hall, London (1999)

2. Armony, M., Ward, A.R.: Fair dynamic routing in large-scale heterogeneous-server systems. Oper. Res. 58(3), 624-637 (2010)

3. Aviv, Y., Federgruen, A.: The value-iteration method for countable state Markov decision processes. Oper. Res. Lett. 24(5), 223-234 (1999)

4. Beutler, F.J., Ross, K.W.: Optimal policies for controlled Markov chains with a constraint. J. Math. Anal. Appl. 112, 236-252 (1985)

5. Chakka, R., Do, T.V.: The $M M \sum_{k=1}^{K} C P P_{k} / G E / c / L G$-queue with heterogeneous servers: steady state solution and application to performance evaluation. Perform. Eval. 64, 191-209 (2007)

6. Ghoneim, H.A., Stidham, S.: Control of arrivals to two queues in series. Eur. J. Oper. Res. 21, 399-409 (1985)

7. Efrosinin, D., Farhadov, M.: Performance analysis and monotone control of a tandem queueing system. DCCN Commun. Comput. Inform. Sci. 279, 241-255 (2014)

8. Efrosinin, D., Rykov, V.: Heuristic solution for the optimal thresholds in a controllable multi-server heterogeneous queueing system without preemption. DCCN Commun. Comput. Inform. Sci. 601, 238-252 (2016)

9. Efrosinin, D., Sztrik, J.: Performance analysis of a two-server heterogeneous retrial queue with threshold policy. Qual. Technol. Quant. Manage. 8(3), 211-236 (2011)

10. Howard, R.: Dynamic Programming and Markov Processes. Wiley, New York (1960)

11. Koole, G.: A simple proof of the optimality of a threshold policy in a two-server queueing system. Syst. Control Lett. 26, 301-303 (1995)

12. Koole, G.: Convexity in tandem queues. Prob. Eng. Inf. Sci. 18(1), 13-31 (2004)

13. Larsen, R.L., Agrawala, A.K.: Control of a heterogeneous two-server exponential queueing system. IEEE Trans. Software Eng. 9(4), 522-526 (1983) 
14. Lehtonen, T.: Stochastic comparisons for many server queues with nonhomogeneous exponential servers. Opsearch 20(1), 1-15 (1983)

15. Liang, H.M., Kulkarni, V.G.: Optimal routing control in retrial queues. In: Shanthikumar, J.G., et al. (eds.) Applied Probability and Stochastic Processes, pp. 203-218. Kluwer Academic Publishers, Boston (1999). Chap. 14

16. Lin, W., Kumar, P.R.: Optimal control of a queueing system with two heterogeneous servers. IEEE Trans. Autom. Control 29, 696-703 (1984)

17. Legros, B., Jouini O.: Routing in a queueing system with two heterogeneous servers in speed and in quality of resolution (2016). http://www.lgi.ecp.fr/ jouini

18. Luh, H.P., Viniotis, I.: Threshold control policies for heterogeneous server systems. Math. Methods Oper. Res. 55, 121-142 (2002)

19. Neuts, M.F.: Matrix-geometric solutions in stochastic models. The John Hopkins University Press, Baltimore (1981)

20. Nobel, R., Tijms, H.C.: Optimal control of a queueing system with heterogeneous servers and set-up costs. IEEE Trans. Autom. Control 45(4), 780-784 (2000)

21. Rosberg, Z., Makowski, A.M.: Optimal routing to parallel heterogeneous servers small arrival rates. Trans. Autom. Control 35(7), 789-796 (1990)

22. Rykov, V.: Monotone control of queueing systems with heterogeneous servers. QUESTA 37, 391-403 (2001)

23. Rykov, V., Efrosinin, D.: On the slow server problem. Autom. Remote Control 70(12), 2013-2023 (2009)

24. Stecke, K., Kim, I.: Performance evaluation for systems of pooled machines of unequal sizes: Unbalancing vs. blocking. European Journal of. Oper. Res. 42, 22 $38(1989)$

25. Özkan, E., Kharoufeh, J.P.: Optimal control of a two-server queueing system with failures. Probab. Eng. Inform. Sci. 28(4), 489-527 (2014)

26. Puterman, M.L.: Markov Decision Process: Discrete Stochastic Dynamic Programming. Wiley, New York (1994)

27. Sennott, L.I.: Stochastic Dynamic Programming and the Control of Queueing Systems. Wiley, New York (1999)

28. Sztrik, J., Roszik, J.: Performance analysis of finite-source retrial queueing systems with nonreliable heterogeneous servers. J. Math. Sci. 146(4), 6033-6038 (2007)

29. Tijms, H.C.: Stochastic Models. An Algorithmic Approach. Wiley, New York (1994)

30. Trancoso, P.: One size does not fit all: a case for heterogeneous multiprocessor systems. In: Proceedings of the IADIS International Conference Applied Computing, Algarve, Portugal (2005)

31. Veatch, M.N., Wein, L.M.: Monotone control of queueing networks. Queueing Syst. 12, 391-408 (1992)

32. Viniotis, I., Ephremides, A.: Extension of the optimality of a threshold policy in heterogeneous multi-server queueing systems. IEEE Trans. Autom. Control 33, 104-109 (1988)

33. Wang, W., Abouzeid, A.A.: Throughput of hybrid radio-frequency and free-spaceoptical (RF/FSO) multi-hop networks. In: Information Theory and Applications Workshop, USA, pp. 1-8, (2007)

34. Weber, R.: On a conjecture about assigning jobs to processors of different speeds. IEEE Trans. Autom. Control 38, 166-170 (1993)

35. Yang, R., Bhulai, S.: Mei., R.: Structural Properties of the Optimal Resource Allocation Policy for Single-Queue Systems. Ann. Oper. Res. 202, 211-233 (2013) 